\title{
Editorial
}

\section{In celebration of the 75th birthday of Professor Anatoly Yagola}

https://doi.org/10.1515/jiip-2020-0134

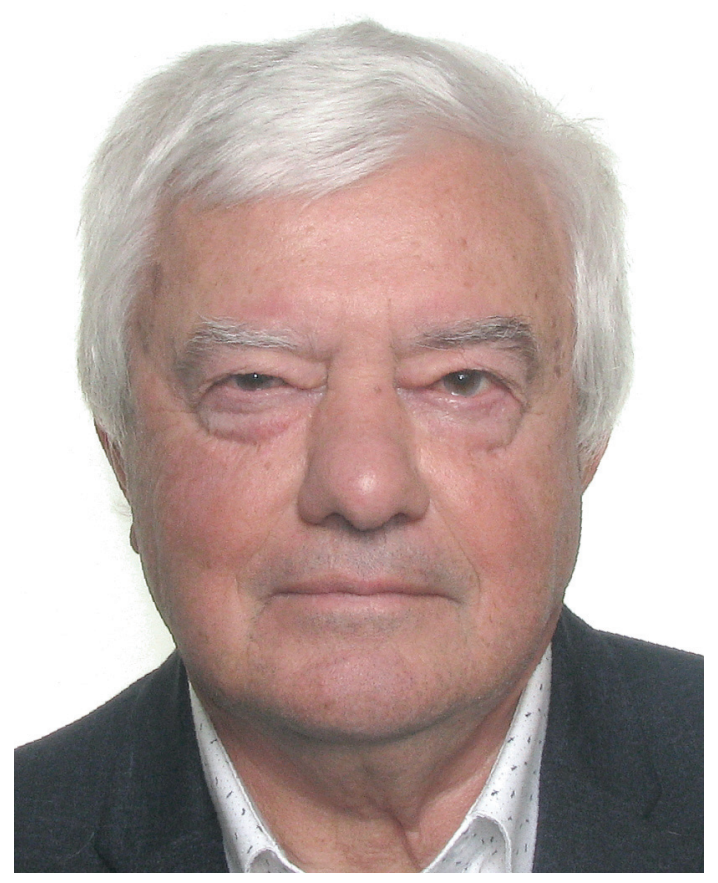

Professor Anatoly Yagola is a distinguished scholar of the inverse problems community and a well-known expert in the field of inverse problems and mathematical modeling.

He was born in 1945 in Moscow, USSR. His father, Grigory Yagola (later Doctor of Technical Sciences, Professor, one of the leading metrologists of USSR), at that time worked as an associate professor at Moscow Power Engineering Institute, and his mother, Yulia Yagola (Lukashenko), was a student at this Institute. In 1962, Anatoly Yagola graduated from the secondary school in Kharkiv and entered the Faculty of Physics of Kharkiv State University. In 1963, his parents moved to the Moscow district, and Anatoly became the second year student of the Faculty of Physics of Lomonosov Moscow State University (MSU). Since then, his entire life was connected with Lomonosov Moscow University.

His scientific adviser was the outstanding mathematician Academician Andrey Tikhonov, to whose scientific school Anatoly Yagola belongs. In 1968 Anatoly became a PhD student. In 1971 Anatoly received the $\mathrm{PhD}$ degree in theoretical and mathematical physics from Lomonosov Moscow State University and started teaching students and doing academic research at the Department of Mathematics of the Faculty of Physics of Lomonosov Moscow State University.

In 1982 Anatoly Yagola completed DrSc dissertation "Ill-posed problems in reflexive spaces", and obtained degree of Doctor of Physics and Mathematics in theoretical and mathematical physics. In 1985 he received the title of Professor.

During many years he served as Vice-Chair of the Scientific and Methodological Council for Mathematics of the Ministry of Education and Science of Russian Federation. He is a member of the American Mathemat- 
ical Society (since 1995), the European Mathematical Society (in 1999-2002 member of the Committee on Applications of Mathematics), and the European Consortium for Mathematics in Industry (1993-2002). He is a founding member of the RAMA network for mathematical analysis in Africa (since 2006). Member of the editorial or advisory boards of the journals: Journal of Inverse and Ill-Posed problems; Inverse Problems in Science and Engineering; Computational Mathematics and Mathematical Physics; Eurasian Journal of Mathematical and Computer Applications; Physics of the Solid Earth; Moscow University Physics Bulletin, and six other journals.

He is Distinguished Professor of Lomonosov Moscow State University and received awards: Award for Young Scientists, Soviet Union (1974); Lomonosov Award (Lomonosov Moscow State University, 1988); Award in Education of the Government of the Russian Federation (2012); the Medal of the Order of Merit "For Services to the Motherland", II class (2017), and others.

Although all works and contributions of Anatoly to inverse problems cannot be mentioned, we should underline that he obtained fundamental results in the theory of linear and nonlinear ill-posed problems in the presence of various a priori information about the solution, and developed effective numerical methods for solving them. In his applications, inverse problems of astrophysics, geophysics, vibrational spectroscopy, electron microscopy, and image processing are considered. As a result, he published more than 300 articles in about twenty mathematical, engineering, and geophysical journals. He is a co-author of about 30 books, including famous monographs:

(1) A. N. Tikhonov, A. V. Goncharsky, V. V. Stepanov and A. G. Yagola, Numerical methods for the solution of ill-posed problems (published in Russian by Nauka, 1990; in English by Kluwer, 1995, and Springer, 2013; in Slovak by Mercury-Smekal Kosice, 2000);

(2) A. N. Tikhonov, A.S. Leonov and A. G. Yagola, Nonlinear ill-posed problems (published in Russian by Nauka, 1995, and KURS, 2017; in English by Chapman and Hall, 1998);

(3) A. G. Yagola, I. V. Kochikov, G. M. Kuramshina and Yu. A. Pentin, Inverse problems of vibrational spectroscopy (published in Russian by MSU publishers, 1993, and KURS, 2017; in English by VSP, 1999, and de Gruyter, 2014);

(4) Y. Wang, I. E. Stepanova, V. N. Titarenko and A. G. Yagola, Inverse problems in geophysics and solution methods (published in Russian by BINOM, 2014, and in Chinese by Higher Education Press Beijing, 2011).

He was a research adviser of more than twenty $\mathrm{PhD}$ students.

He has brought together numerous members of Russian community by organizing the permanent (from 1974) scientific seminar on inverse problems in mathematical physics. He took part in organization of many international workshops and conferences.

On behalf of colleagues, students, and friends from all over the world, we would like to wish Anatoly on the occasion of his 75 th birthday every success in scientific work and much happiness with his family. Anatoly is very active in his research and teaching covering various important topics in applied mathematics. He plays a leading role in the inverse problems community. He is also a very nice and friendly person.

O. Alifanov, Moscow Aviation Institute, Russia

L. Beilina, Chalmers University of Technology and Gothenburg University, Sweden

Dinh Nho Hao, Institute of Mathematics, Vietnam

G. Dulikravich, Florida International University, USA

H. Engl, University of Vienna, Austria

A. Hasanov, Kocaeli University, Turkey

B. Hofmann, Chemnitz University of Technology, Germany

S. Kabanikhin, Institute of Computational Mathematics and Mathematical Geophysics SB RAS, Russia

M. Klibanov, University of North Carolina Charlotte, USA

G. Kuramshina, Lomonosov Moscow State University, Russia

D. Lesnic, University of Leeds, UK

D. Lukyanenko, Lomonosov Moscow State University, Russia

Z. Nashed, University of Central Florida, USA 
N. Nefedov, Lomonosov Moscow State University, Russia

T. Reginska, Institute of Mathematics, Poland

V. Romanov, Sobolev Institute of Mathematics, Russia

V. Sadovnichy, Lomonosov Moscow State University, Russia

O. Scherzer, University of Vienna, Austria

C. Sebu, University of Malta

M. Shishlenin, Institute of Computational Mathematics and Mathematical Geophysics SB RAS, Russia

A. Shkalikov, Lomonosov Moscow State University, Russia

Y. Sultanaev, Bashkir State Pedagogical University, Russia

A. Tikhonravov, Lomonosov Moscow State University, Russia

E. Tyrtyshnikov, Marchuk Institute of Numerical Mathematics, Russia

V. Vasin, Institute of Mathematics and Mechanics, Russia

Y. Wang, Institute of Geology and Geophysics, China

M. Yamamoto, University of Tokyo, Japan 